\title{
Fabrication of Light Extraction Efficiency of Organic Light-Emitting Diodes with 3D Aspherical Microlens by Using Dry Etching Process
}

\author{
Y. C. Chen, ${ }^{1}$ C. T. Pan, ${ }^{1,2}$ C. C. Hsieh, ${ }^{3}$ C. Y. Su, ${ }^{4}$ H. C. Wu, ${ }^{1}$ and W. C. Li $^{1}$ \\ ${ }^{1}$ Department of Mechanical and Electro-Mechanical Engineering, National Sun Yat-Sen University, Kaohsiung 80424, Taiwan \\ ${ }^{2}$ Center for Nanoscience \& Nanotechnology, National Sun Yat-Sen University, National Science Council Core Facilities Laboratory for \\ Nano-Science and Nano-Technology in Kaohsiung-Pingtung Area, Taiwan \\ ${ }^{3}$ Department of the Mechanical and Automation Engineering, National Kaohsiung First University of Science and Technology, \\ Kaohsiung 811, Taiwan \\ ${ }^{4}$ Institute of Manufacturing Technology, National Taipei University of Technology, Taipei 106, Taiwan
}

Correspondence should be addressed to C. C. Hsieh; cchsieh@nkfust.edu.tw

Received 13 September 2013; Accepted 11 October 2013

Academic Editor: Liang-Wen Ji

Copyright (c) 2013 Y. C. Chen et al. This is an open access article distributed under the Creative Commons Attribution License, which permits unrestricted use, distribution, and reproduction in any medium, provided the original work is properly cited.

organic light-emitting diode (OLED) can enable a greater artificial contrast ratio and viewing angle compared to liquid crystal display (LCD) because OLED pixels directly emit light. There is a shortcoming that the internal quantum efficiency can reach values close to $100 \%$, but about $80 \%$ light disperses because of the difference among the refractive indices of the substrate, anode, indium tin oxide (ITO) film, and air. In this paper, three dimensions aspherical microlens arrays (3D A-MLAs) with substrate modifications are developed to simulate the optical luminous field by using FRED software. This study modified parameters of 3D A-MLAs such as the diameter, fill-factor, aspect ratio, dry etching parameters, and electroforming rates of microlens to improve the extraction efficiency of the OLED. In dry etching, not only the aspect ratio with better extraction rate can be obtained by reactive ion etching (RIE) dry etching, but also an undercutting phenomenon can be avoided. The dimensions of 3D A-MLAs can be accurately controlled in the electroforming process used to make a nickel-cobalt ( $\mathrm{Ni}-\mathrm{Co})$ metal mold to achieve the designed dimensions. According to the measured results, the average luminance efficacy of the OLEDs with 3D A-MLAs can be enhanced.

\section{Introduction}

In this rapid-growing information era, display has become an indispensable information tool. In the flat panel displays (FPDS), the organic light-emitting diode (OLED) display is attracting considerable interest, because it is much thinner, lighter, and more flexible than liquid crystal display (LCD).

OLED seems to be the perfect technology for all types of displays, but the extraction efficiency of current OLED is generally low due to its total internal reflection, and the internal quantum efficiency is limited because light disperses in different refractive indices [1-3]. Thus, how to increase the OLED extraction efficiency of luminance is worth studying. The extraction efficiency of luminance could be increased by many methods, such as using the resonant cavity [4], excitation of surface plasmons [5], insertion of a thin silica aerogel layer of very low refractive index [6], and $2 \mathrm{D}$ $\mathrm{SiO}_{2} / \mathrm{SiN}_{x}$ photonic crystals [7] as well as microlens arrays [8]. Among these technologies, the process of microlens arrays is simple and reliable, so this study analyzed the manufacturing and designed parameters of microlens arrays (MLAs). The luminance will be increased as the contact angle and fill-factor of MLA [9] increase, and the luminance will be decreased as the viewing angle is increases [10].

Being a commercial fabricating process, it should be of low cost and rapid. But the conventional methods of fabricating A-MLAs such as gray-scale mask, sandblasted diffuser plate, and lithography with SU-8 still have some 
disadvantages [11-13]. Those processes need highly accurate alignment and control, long process time, high production cost, and special material. To solve these problems, we presented a modified LIGA process. A metal mold is applied to replicate MLA rapidly by using this method, and AMLAs could be produced with any shape. So far, hexagonal [14], triangular [15], and dual-curvature MLAs [1] were successfully fabricated accurately in our laboratory.

The aspherical microlens shape attached on the OLED can reduce the ratio of total reflection in substrate to improve the efficiency of an OLED when the waveguide phenomenon which occurs between air and glass is reduced. three dimensions aspherical microlens arrays (3D A-MLAS) optical films were fabricated. In electroforming, the Ni-Co alloy was deposited on the seed layer, and it forms a metal mold that is also called the first mold. The second mold was fabricated by polydimethylsiloxane (PDMS), which was replicated from the first mold. The parameters of the process such as diameter of MLA, dry etching, and electroforming rates were analyzed. We used SpectraScan Colorimeter PR650 to measure the luminance efficiency. The luminance of OLEDs can be enhanced significantly.

\section{Materials and Methods}

2.1. Design and Simulation. The fill-factor is the ratio of the active refracting area to the total contiguous area occupied by the lens array. And the aspect ratio is the proportional relationship between width and height of MLA. This study used the optical simulation software, FRED, to analyze the variations of the optical characteristics such as the diameter and aspect ratio. This analysis is based on the theory that when the aspect ratios of A-MLAs are higher, the intensity of a light source is higher. The parameters include curves, four layouts, and four diameters. The variations of intensity with respect to diameters are limited in the same layout with different diameters. In the design, fill-factor and aspect ratio are two important factors. The higher fill-factor and aspect ratio an MLA has, the higher extraction rate can be obtained. These experiments focus on how the different parameters of dry etching and electroforming rate influence the formation of A-MLAs with the same diameters. Figure 1 is a gain of luminous intensity of OLED with MLAs film with a spherical curve in different layouts. The simulation results show that luminous intensity of the layout of tangential circle is obviously higher than of the other layouts. For the same aspect ratio of 0.5 , the luminous intensity can be enhanced up to $28 \%$ which is compared with a bare OLED light source without optical film. Due to the geometric constraints, the maximum aspect ratio of a spherical MLA is 0.5 . In order to collect the maximum amount of light, the lens should be as close to $100 \%$ as possible [16].

2.2. Fabrication and Process. The study develops 3D AMLAs by modified lithographie, galvanoformung, abformung (LIGA) process. The procedure is shown in Figure 2.

(1) Dehydrate RCA cleaning removes the organic contaminants, thin oxide layer, and ionic contamination on wafers.

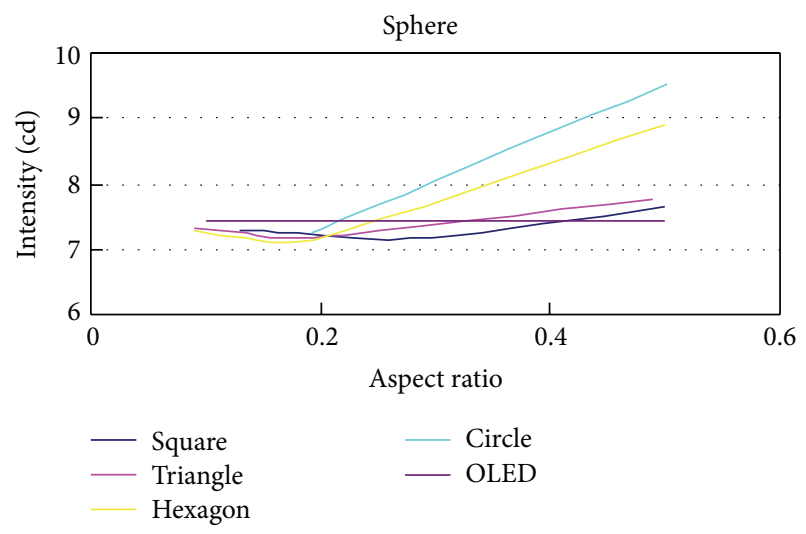

FIgURE 1: A gain of luminous intensity of OLED with MLAs film with a spherical curve in different layouts.

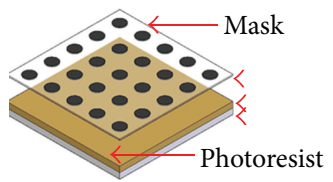

(a)

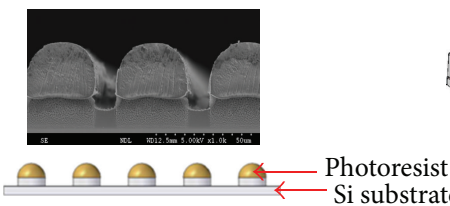

(c)

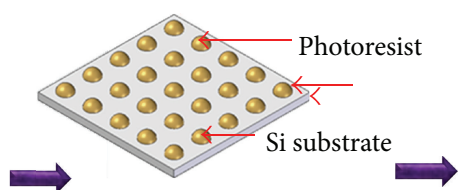

(b)

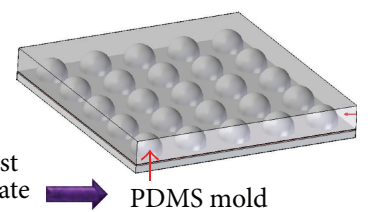

(d)
FIGURE 2: The procedure of the modified LIGA process which can manufacture an A-MLA with high aspect ratio (a) exposure process, (b) reflow process, (c) RIE dry etching process, and (d) PDMS mold.

(2) Spinning positive photoresist (PR), AZ4620, on wafers.

(3) After soft baking, the PR is exposed to a pattern of intense light, as shown in Figure 2(a). The exposure to light causes a chemical change that makes the PR become soluble in the developer when it is exposed, and the pitch and dimensions of mask pattern were $70 \mu \mathrm{m}$ and $40 \mu \mathrm{m}$, respectively, which should be taken into consideration carefully to manufacture an AMLA.

(4) Reflow process can make a column array become a microlens array, as shown in Figure 2(b). We used reactive ion etching (RIE) dry etching to avoid significant undercutting of photoresist pattern, as shown in Figure 2(c). In dry etching, different concentrations of oxygen can obviously influence the shape and surface roughness of microstructures.

Sputtering silver $(\mathrm{Ag})$ layer as a seed layer on the wafer then depositing $\mathrm{Ni}$-Co alloy as an electroforming mold on it is called first mold. In electroforming, various ampere per square decimeter (ASD) $\left(\mathrm{A} / \mathrm{dm}^{2}\right)$ can influence deposition rate and roughness and then replicate the first mold by PDMS 
TABLE 1: The parameters of RIE.

\begin{tabular}{lcccccc}
\hline No. & Pressure $(\mathrm{m}$ Torr $)$ & $\mathrm{O}_{2}(\mathrm{sccm})$ & Time $($ minutes $)$ & Diameter $(\mu \mathrm{m})$ & Height $(\mu \mathrm{m})$ & Aspect ratio \\
\hline 1 & 25 & 10 & 10 & 36.58 & 31.76 & 0.45 \\
2 & 25 & 5 & 10 & 30.83 & 21.83 & 0.68 \\
3 & 25 & 1 & 10 & 30.65 & 22.57 & 22.80 \\
4 & 25 & 0 & 10 & 0.73 \\
\hline
\end{tabular}

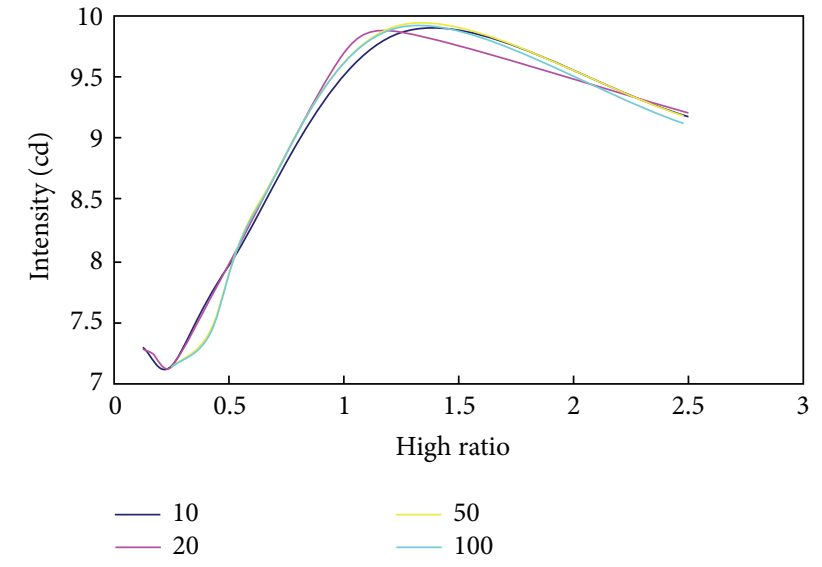

FIGURE 3: The simulated results of comparison of luminous intensity with different diameters and aspect ratios.

TABLE 2: The dimensions of A-MLAs on a film.

\begin{tabular}{lccc}
\hline & Diameter $(\mu \mathrm{m})$ & Height $(\mu \mathrm{m})$ & Aspect ratio \\
\hline Bare light & NA & NA & NA \\
Low-ASD film & 67.45 & 30 & 0.45 \\
High-ASD film & 70.02 & 30 & 0.43 \\
NE-film & 33.71 & 25 & 0.72 \\
\hline
\end{tabular}

$[17,18]$, as shown in Figure 2(d). Finally, the optical film with A-MLAs by ultraviolet (UV) cured process was fabricated.

\section{Results and Discussion}

3.1. Dimensions of A-MLAs. The diameters of 3D microlenses are set as $10,20,50$, and $100 \mu \mathrm{m}$ modified LIGA process, and the aspect ratios are from 0.1 to 2.5 . While the aspect ratio is larger than 1.25 , the luminous intensity decreases gradually (Figure 3). The height of a microlens can be achieved by the etching and electroforming process.

3.2. Diameters of A-MLAs. Comparing the diameter of 3D AMLAs simulated by FRED with the diameter manufactured by UV cured process, the radius decreased from $70 \mu \mathrm{m}$ to $69.4 \mu \mathrm{m}$. The substrate was exposed to UV light. After developing and thermal reflow, a microcolumn array was obtained, and the diameter was $38.4 \mu \mathrm{m}$. This error was $4 \%$. Then we used RIE dry etching to avoid significant undercutting of photoresist pattern, and the diameter was $36.3 \mu \mathrm{m}$. This error was $5.5 \%$. After dry etching and electroforming process, a microlens array was cast by using PDMS process, and the diameter was $69.5 \mu \mathrm{m}$. This error was $0.7 \%$.

3.3. Dry Etching Parameters. RIE with incident oxygen $\left(\mathrm{O}_{2}\right)$ plasma and sulfur hexafluoride $\left(\mathrm{SF}_{6}\right)$ was performed to shrink the size of A-MLAs in order to facilitate the etching of photoresist. The RIE was performed on spheres with variable $\mathrm{O}_{2}$ flow rates $(10,5,1$, and $0 \mathrm{sccm})$. The etching parameter is shown in Table 1 . The aspect ratio became worse as the height which was $16.8 \mu \mathrm{m}$ of A-MLAs was reduced obviously.

The flow rate was set to be $10 \mathrm{sccm}$ with a chamber pressure of $25 \mathrm{mTorr}$ and the power of $40 \mathrm{~W}$ in the first experiment, as shown in Figures 4(a) and 4(b). The heights were 21.9 and $22.6 \mu \mathrm{m}$ in the second and third experiments, as shown in Figures 4(c) and 4(d), and the etching rates were $1: 1.85$ and $1: 2.1$ separately.

Scanning electron microscopic (SEM) images of a 3D A-MLA without $\mathrm{O}_{2}$ dry etching which could not be used because of its low etching efficiency. The study found that the lower $\mathrm{O}_{2}$ concentration is, the much higher and better shape could be obtained, but if $\mathrm{O}_{2}$ concentration was too low (below $1 \mathrm{sccm}$ ) as the seventh experiment, the gas would damage the structure seriously, so the concentration should be well controlled.

3.4. Electroforming Rates. Photoresist surface has many defects after RIE etching, and the dimensions are shrunk by etching. Electroforming process can smooth the surface defects by depositing metal layers to replicate PDMS molds. During the electroplating, the lens grows isotropically. The development of a hexagonal microlens array is influenced by the gaps between etching patterns and the diameters of microlenses (Figure 5(a)). Furthermore, the electroplating technology is an important process to fabricate the hexagonal shape. Then, a Ni-Co alloy was cast atop the seed layer, and it formed a microgapless hexagonal microlens array (Figure 5(b)). Therefore the profile of hexagonal lens is spherical, same as the microlens of PR. The metal mold with higher ASD is better than the one with lower ASD, as shown in Figure 6.

3.5. Measurement of the Film Pasted on an OLED. The luminance, current density, and luminance efficiency tested by SpectraScan Colorimeter PR-650 (Photo Research, Inc.) were controlled by a computer and a power supply. The power supply (2400 Source Meter, Keithley) can provide direct current (DC) to an OLED as a light source in this measurement. 


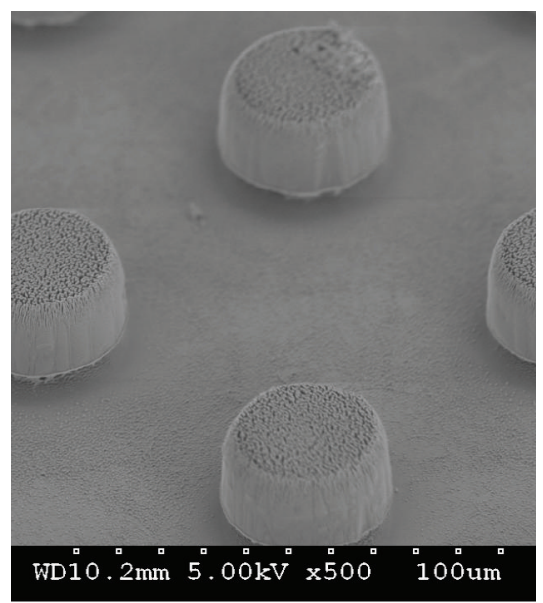

(a)

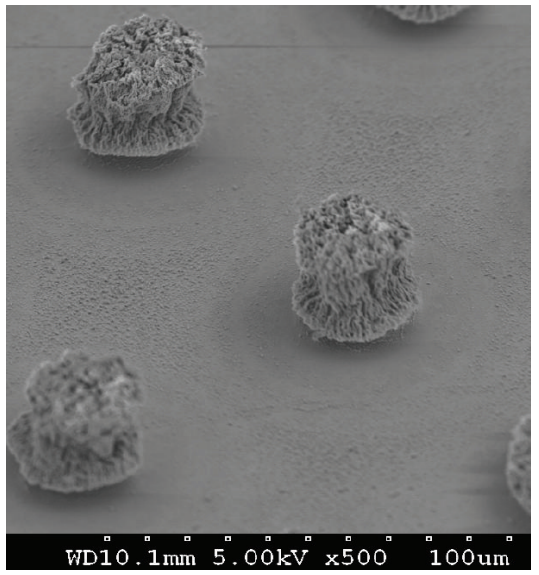

(c)

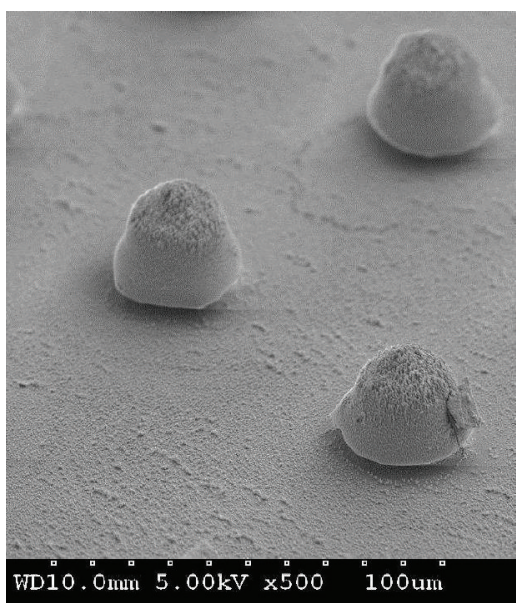

(b)

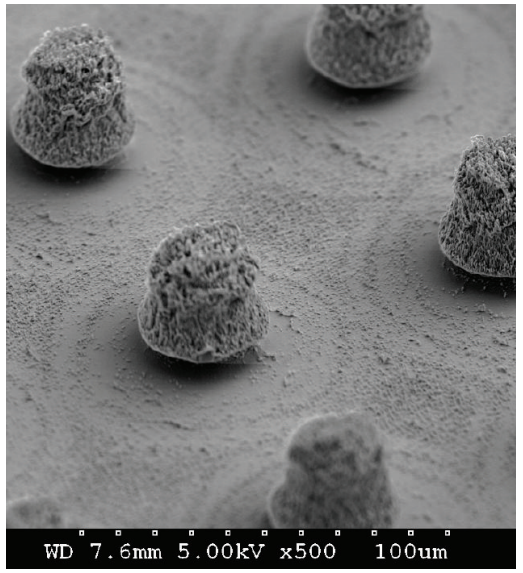

(d)

Figure 4: The results with different parameters of dry etching process: (a) the 1st experiment with oxygen concentration of $10 \mathrm{sccm}$, (b) the 2nd experiment with oxygen concentration of $5 \mathrm{sccm}$, (c) the $3 \mathrm{rd}$ experiment with oxygen concentration of $1 \mathrm{sccm}$, and (d) the 4 th experiment without oxygen.

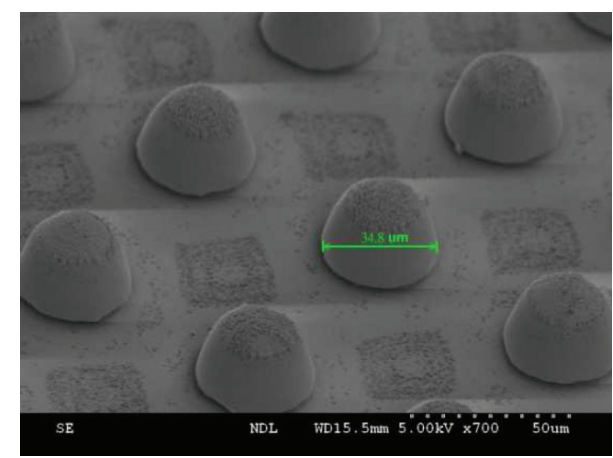

(a)

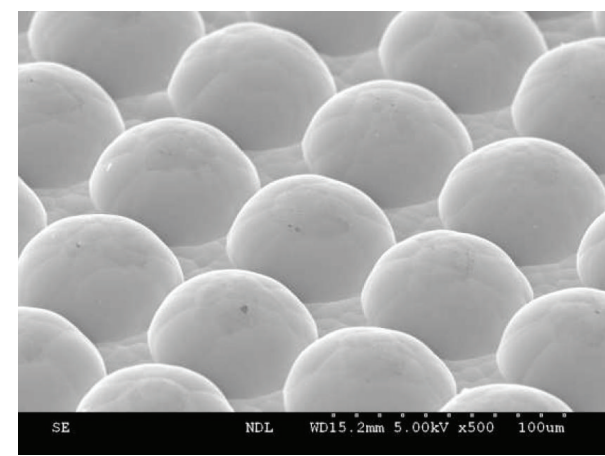

(b)

FIGURE 5: (a) SEM image of a mold of microlens array; (b) SEM image of a mold of hexagonal microlens array.

TABLE 3: The measured data of each film.

\begin{tabular}{lcccc}
\hline & Base-light & High-ASD film & Low-ASD film & NE-film \\
\hline Relative ratio (compared to Base-light) & 1 & 15.1 & 12.5 & 5.7 \\
\hline
\end{tabular}




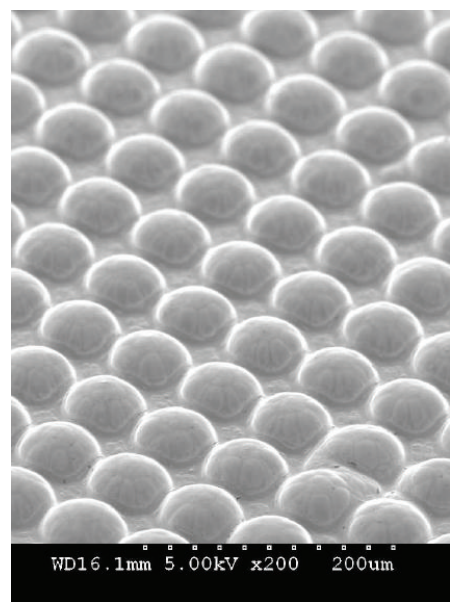

(a)

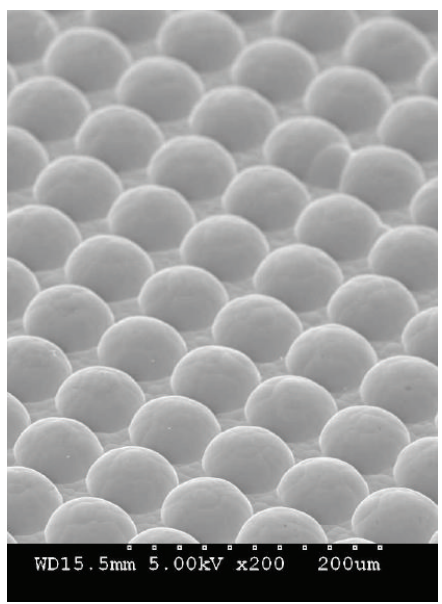

(b)

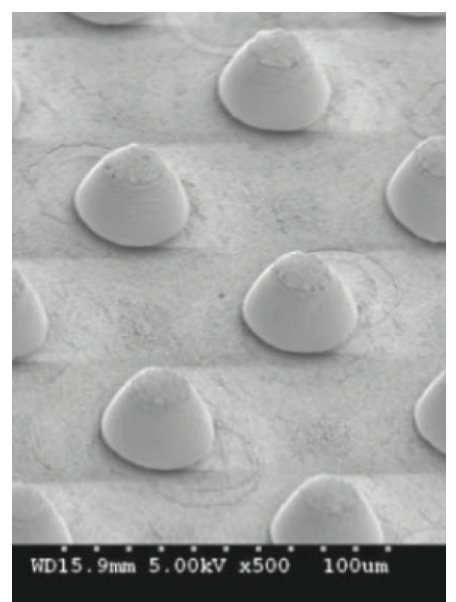

(c)

FIGURE 6: The SEM results of the electroforming experiment: (a) electroforming with low ASD; (b) electroforming with high ASD; and (c) dry etching without electroforming.

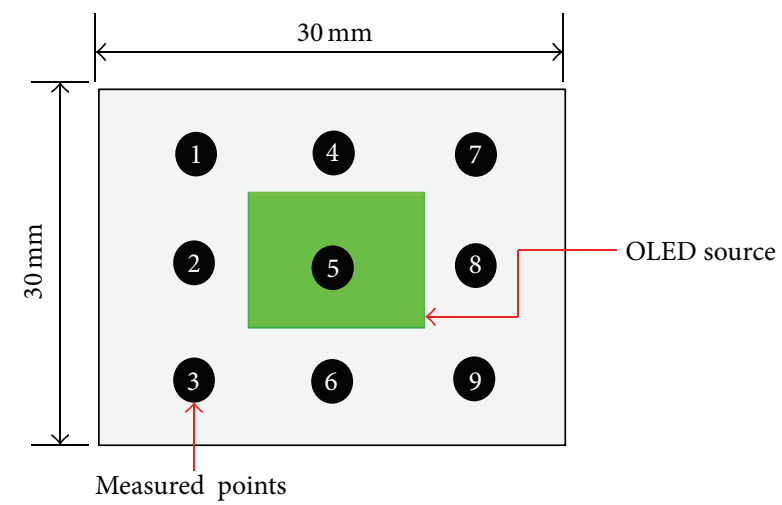

FIGURE 7: Nine-point measurement.

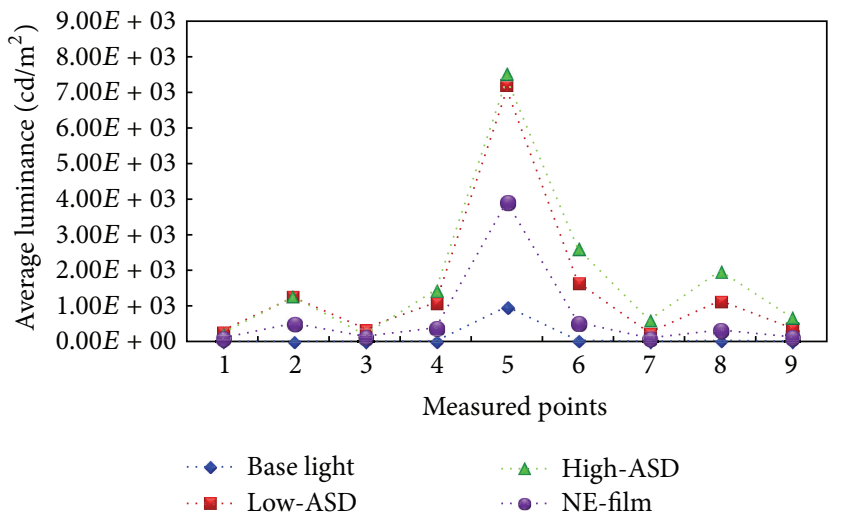

FIGURE 8: The average luminance of four measurements.

For the measurement of A-MLAs, four samples including bare light, A-MLAs with low ASD called low-ASD film, AMLAs with high ASD called high-ASD film, and an etched optical film without electroforming were used to generate and select suitable items called NE-film (Table 2). The measured

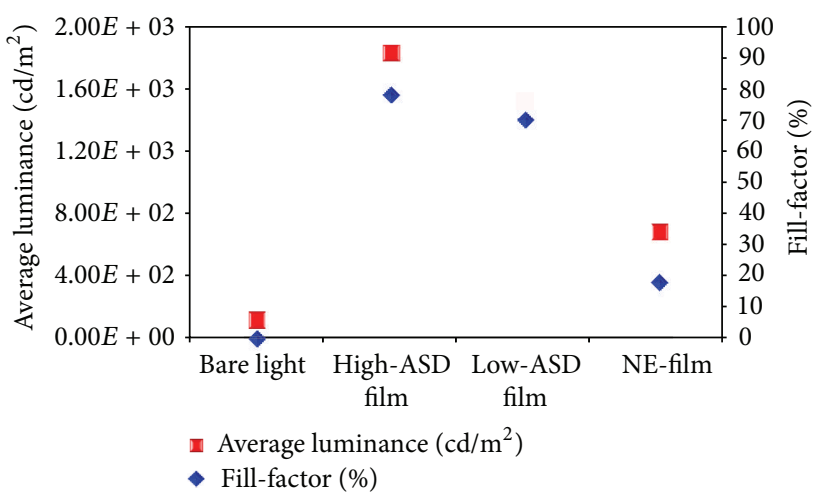

FIGURE 9: The comparisons of ratios of three optical films and one light source.

data of each film is shown Table 3 is the all calculated results. Comparison between bare light and an OLED with films is obtained, and the largest variation is 15 times the luminance of the bare light.

The luminance and uniformity were measured by ninepoint measurement (Figures 7, 8, and 9), and the optical film was attached to an OLED. The measurement of the bare light was used as base data to compare with the others, and the optical film with A-MLAs was 15 times brighter than bare light.

\section{Conclusion}

After dry etching process was applied into the LIGA-like process, aspect ratio of 3D A-MLA was improved significantly. By replicating PDMS mold rapidly, the time-wasted microelectroforming process could be removed. There are several advantages in our study. 
(1) 3D A-MLAs with high aspect ratio could be fabricated by using modified LIGA process that combined reflow process and dry etching.

(2) By using electroforming technology, 3D A-MLA with high fill-factor could be achieved.

(3) The time of process and cost could be reduced by applying the PDMS replicating and UV curing technologies to the process.

(4) After 3D A-MLAs were used, the luminance of OLED could be enhanced significantly, and the efficiency of light source was raised as well.

\section{Acknowledgments}

The authors like to thank F. T. Hsu, P. H. Lin, and the National Sun Yat-Sen University for the assistance in this project.

\section{References}

[1] V. Bulović, V. B. Khalfin, G. Gu, P. E. Burrows, D. Z. Garbuzov, and S. R. Forrest, "Weak microcavity effects in organic lightemitting devices," Physical Review B, vol. 58, no. 7, pp. 37303740, 1998.

[2] G. Nenna, D. Mauro, A. De Girolamo Del Mauro et al., "Optical properties of polystyrene- $\mathrm{ZnO}$ nanocomposite scattering layer to improve light extraction in organic light-emitting diode," Journal of Nanomaterials, vol. 2012, Article ID 319398, 7 pages, 2012.

[3] W. Xu and Y. Li, "The effect of anisotropy on light extraction of organic light-emitting diodes with photonic crystal structure," Journal of Nanomaterials, vol. 2013, Article ID 969120, 6 pages, 2013.

[4] T. Shiga, H. Fujikawa, and Y. Taga, "Design of multiwavelength resonant cavities for white organic light-emitting diodes," Journal of Applied Physics, vol. 93, no. 1, pp. 19-22, 2003.

[5] N. N. Dinh, D. N. Chung, T. T. Thao, and D. Hui, "Study of nanostructured polymeric composites used for organic light emitting diodes and organic solar cells," Journal of Nanomaterials, vol. 2012, Article ID 190290, 6 pages, 2012.

[6] T. Tsutsui, M. Yahiro, H. Yokogawa, K. Kawano, and M. Yokoyama, "Doubling coupling-out efficiency in or-ganic lightemitting devices using a thin silica aerogel layer," Advanced Materials, vol. 13, no. 15, pp. 1149-1152, 2001.

[7] Y. R. Do, Y. C. Kim, Y.-W. Song et al., "Enhanced light extraction from organic light-emitting diodes with $2 \mathrm{D} \mathrm{SiO}_{2} / \mathrm{SiN}_{x}$ photonic crystals," Advanced Materials, vol. 15, no. 14, pp. 1214-1218, 2003.

[8] S. Möller and S. R. Forrest, "Improved light out-coupling in organic light emitting diodes employing ordered microlens arrays," Journal of Applied Physics, vol. 91, no. 5, pp. 3324-3327, 2002.

[9] Y. Sun and S. R. Forrest, "Organic light emitting devices with enhanced outcoupling via microlenses fabricated by imprint lithography," Journal of Applied Physics, vol. 100, no. 7, Article ID 073106, 2006.

[10] M.-K. Wei, J.-H. Lee, H.-Y. Lin et al., "Efficiency improvement and spectral shift of an organic light-emitting device by attaching a hexagon-based microlens array," Journal of Optics A, vol. 10, no. 5, Article ID 055302, 2008.
[11] B. H. Cumpston, I. D. Parker, and K. F. Jensen, "In situ characterization of the oxidative degradation of a polymeric light emitting device," Journal of Applied Physics, vol. 81, no. 8, pp. 3716-3720, 1997.

[12] S.-I. Chang, J.-B. Yoon, H. Kim, J.-J. Kim, B.-K. Lee, and D. H. Shin, "Microlens array diffuser for a light-emitting diode backlight system," Optics Letters, vol. 31, no. 20, pp. 3016-3018, 2006.

[13] J.-N. Kuo, C.-C. Hsieh, S.-Y. Yang, and G.-B. Lee, "An SU8 microlens array fabricated by soft replica molding for cell counting applications," Journal of Micromechanics and Microengineering, vol. 17, no. 4, pp. 693-699, 2007.

[14] C. Adachi, M. A. Baldo, M. E. Thompson, and S. R. Forrest, "Nearly $100 \%$ internal phosphorescence efficiency in an organic light emitting device," Journal of Applied Physics, vol. 90, no. 10, pp. 5048-5051, 2001.

[15] M. Ikai, S. Tokito, Y. Sakamoto, T. Suzuki, and Y. Taga, "Highly efficient phosphorescence from organic light-emitting devices with an exciton-block layer," Applied Physics Letters, vol. 79, no. 2, pp. 156-158, 2001.

[16] C. T. Pan, Y. C. Chen, M. F. Chen, and Y. C. Hsu, "Fabrication and design of various dimensions of multi-step ashperical microlens arrays for OLED package," Optics Communications, vol. 284, no. 13, pp. 3323-3330, 2011.

[17] W. M. Choi and O. O. Park, "A soft-imprint technique for submicron-scale patterns using a PDMS mold," Microelectronic Engineering, vol. 73-74, pp. 178-183, 2004.

[18] T. Fujii, "PDMS-based microfluidic devices for biomedical applications," Microelectronic Engineering, vol. 61-62, pp. 907914, 2002. 

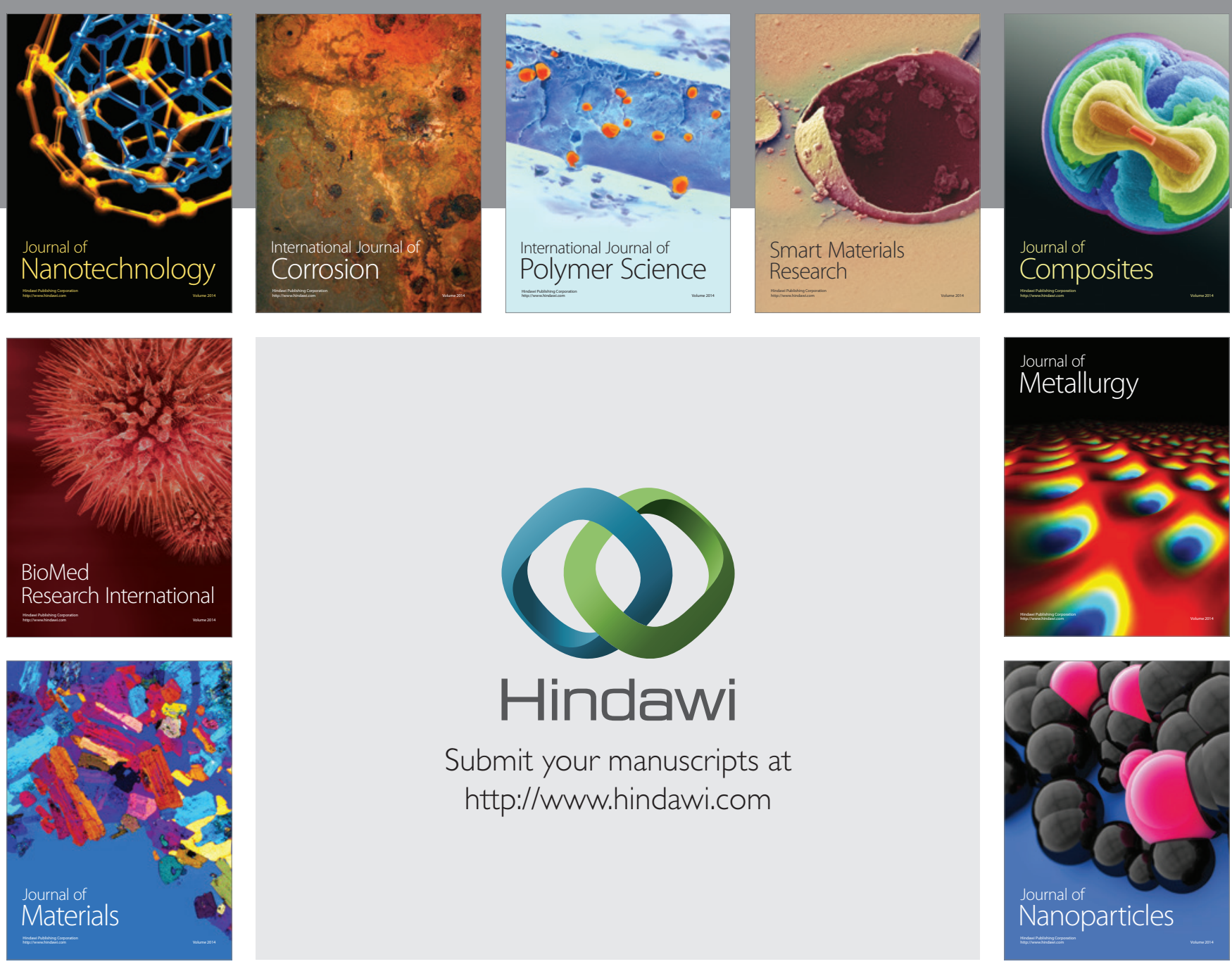

Submit your manuscripts at http://www.hindawi.com
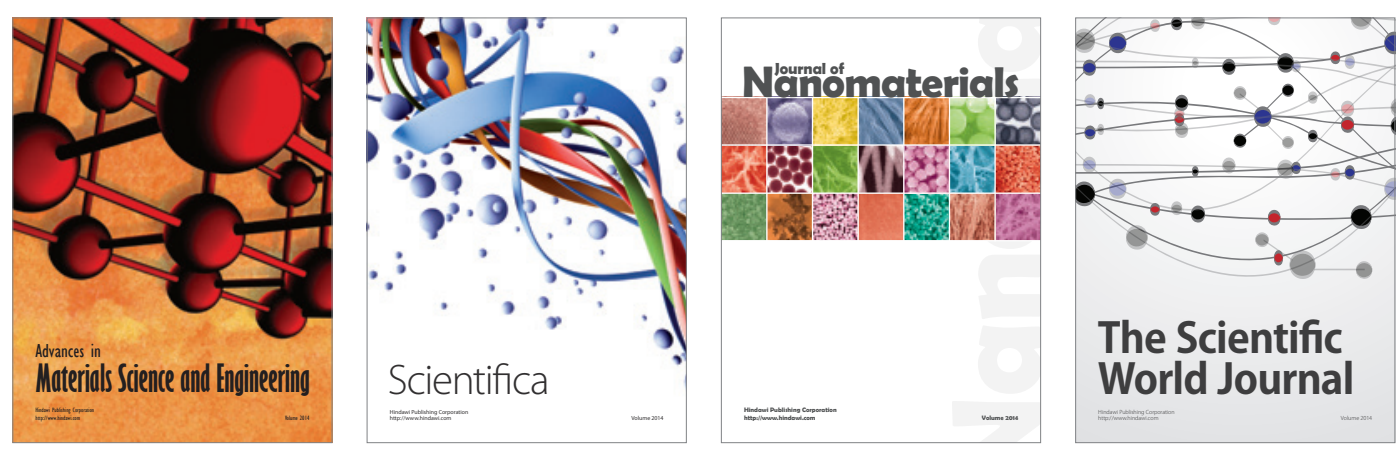

\section{The Scientific World Journal}
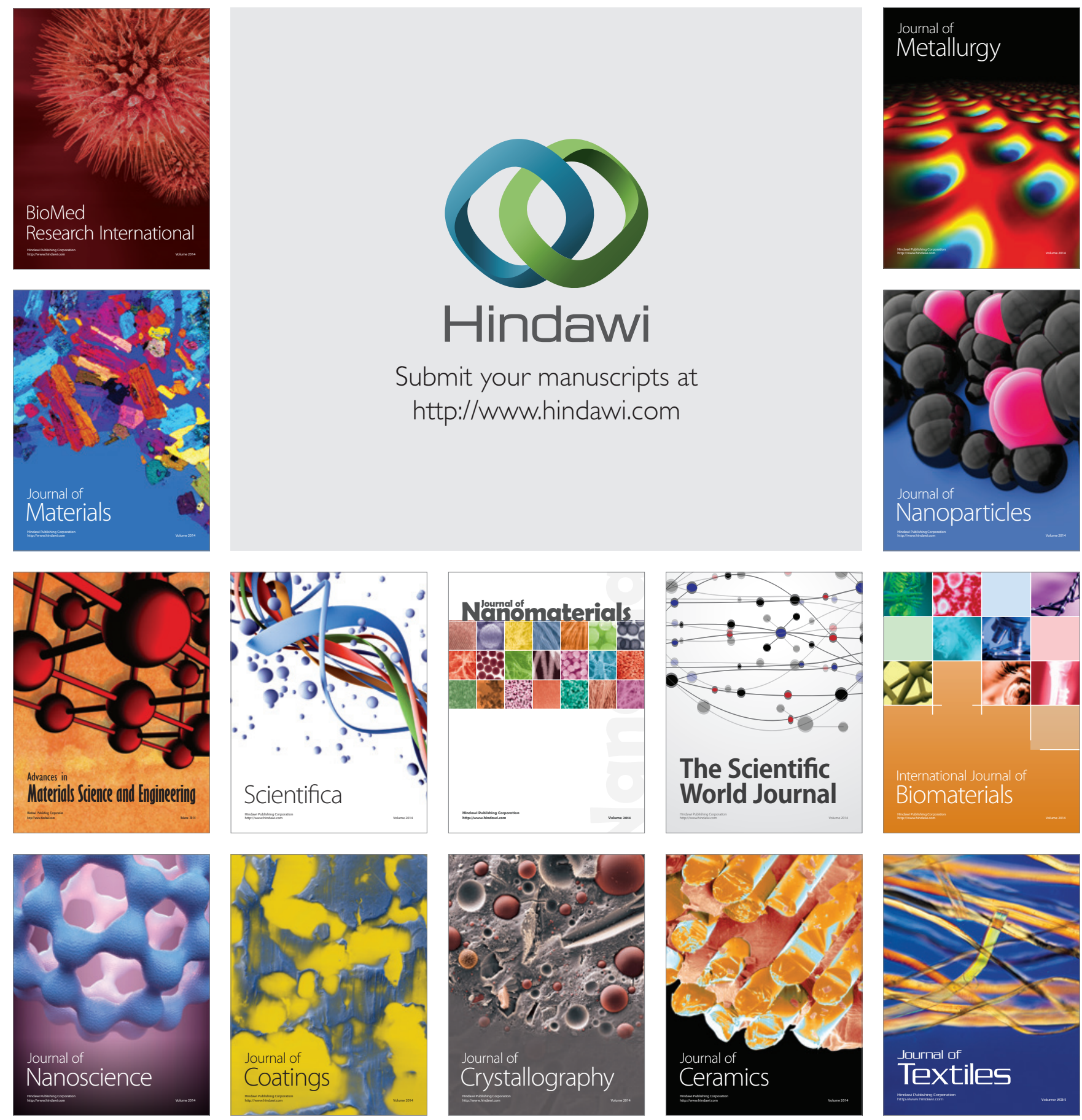